\title{
Análisis de las concepciones pedagógicas y comunicativas de un curso MOOC
}

\section{Mariana Lima ${ }^{\mathrm{a}}$, Elia Fernández Díaz ${ }^{\mathrm{b}}$ y Carlos Rodríguez Hoyos $^{\mathrm{c}}$}

aUniversidad de Cantabria, delimam@unican.es,bfdiazem@unican.es y ccarlos.rodriguezh@unican.es.

\begin{abstract}
This work is carried out through the European project for the creation and development of MOOCs (ECO: E-learning and Open Data Comunication: Masive, Mobile, Ubiquitous and Open Learning). This work aims to analyze the educational and communication conceptions in the design and development of a MOOC "Educational Innovation and Professional Development. Possibilities and limits of ICT". The results show the effectiveness of a critical curricular approach in the design of this course, as well as enhancement of a collaborative work environment, as major achievements. From the difficulties encountered, new alternatives to improve future editions of course have been proposed.
\end{abstract}

Keywords: Elearning, MOOC, pedagogical model, communicative model

\section{Resumen}

Este trabajo se desarrolla en el marco de un proyecto europeo para la creación y desarrollo de MOOCs (ECO: E-learning Comunication and Open Data: Masive, Mobile, Ubiquitous and Open Learning). El trabajo tiene como objetivo analizar las concepciones pedagógicas y comunicativas en el diseño y desarrollo de un curso MOOC "Innovación Educativa y Desarrollo Profesional. Posibilidades y límites de las TIC". Los resultados ponen de manifiesto la eficacia de la negociación de un enfoque crítico curricular en el diseño del curso, así como la potenciación de un entorno de trabajo colaborativo, como principales logros. A partir de las dificultades detectadas, se plantean nuevas alternativas para la mejora en las próximas ediciones del curso.

Palabras clave: Elearning, MOOC, modelo pedagógico, modelo comunicativo 
Análisis de las concepciones pedagógicas y comunicativas de un curso MOOC

\section{Introducción}

En este trabajo presentamos un análisis del diseño y desarrollo de un MOOC sobre innovación educativa a través de las TIC.

Este curso se ha llevado a cabo en el marco de un proyecto, dirigido a la creación y desarrollo de MOOCs, titulado "Elearning Communication Open-Data: Massive Mobile, Ubiquitous and Open Learning (ECO)", financiado por la Unión Europea a través del "Competencies and Innovation Framework Programme" (CIP). El proyecto se orienta a diseñar, desarrollar y evaluar diferentes MOOCs destinados a formadores y profesores de todos los niveles educativos con el objeto de proporcionarles herramientas básicas tanto para el desarrollo de este tipo de curso, a través de las tecnologías móviles, como para especializarse en diferentes ámbitos en función de la temática del curso (innovación educativa, exclusión digital, entre otros). Dicho proyecto está coordinado por la UNED y participan en el mismo diferentes instituciones universitarias y empresas.

A partir de una amplia revisión bibliográfica sobre las concepciones pedagógicas y comunicativas de los MOOCs se establece un marco común de actuación orientado a plantear un modelo alternativo de diseño de cursos. Fundamentalmente, dicha visión subraya la necesidad de potenciar modelos de comunicación horizontales y participativos, utilizando diferentes herramientas para promover un diálogo intercultural a nivel global y fomentar la concienciación social (Dezuanni y Monroy-Hernandez, 2012; Tapscott, 2009). En este sentido, algunas experiencias analizadas alertan sobre la necesidad de repensar el enfoque subyacente al diseño de estos entornos para favorecer el trabajo en red y la creación de itinerarios personales (Gea y Montes, 2013; Flores et al., 2013, Montero y Viñuales, 2013).

Tal y como se ha puesto de manifiesto en trabajos anteriores (Fernández, Rodríguez y Fueyo, 2014), el análisis realizado sobre las concepciones pedagógicas y comunicativas de los MOOCs ha permitido establecer una serie de directrices para orientar el diseño y desarrollo de dichos cursos, con el objeto de garantizar experiencias innovadoras. Por un lado, a nivel pedagógico, es preciso abandonar el modelo tecnológico para garantizar la colaboración de los profesionales que intervienen y la negociación en el establecimiento de las metas así como superar las limitaciones curriculares inherentes a determinados tipos de evaluación centradas únicamente en el producto, tratando de incorporar alternativas (O’Toole, 2013). Por otro lado, a nivel comunicativo, se requiere partir de un modelo capaz de facilitar la recepción crítica y la producción creativa de la información, huyendo de un modelo de participante como mero consumidor de información (Cloutier, 2010) y asegurando el establecimiento de interacciones y conexiones significativas (Mackness, Mark y Williams, 2010). 
En este marco de trabajo se ha diseñado y desarrollado el curso "Innovación educativa y desarrollo profesional. Posibilidades y límites de las TIC" cuyo análisis acometeremos en esta comunicación.

\section{Objetivos}

Con el propósito de lograr la finalidad esencial de la experiencia, esto es, analizar las concepciones pedagógicas y comunicativas inherentes al diseño y desarrollo de un MOOC, se plantean los siguientes objetivos específicos:

- Analizar bibliográficamente, desde un marco pedagógico y comunicativo, los requisitos y características de las plataformas MOOC.

- Generar un entorno de trabajo idóneo para el diseño, implementación y evaluación del MOOC.

- Seleccionar las herramientas y recursos implicados en el diseño de los diferentes elementos curriculares del proceso de enseñanza-aprendizaje.

- Negociar una dinámica de trabajo colaborativa para fomentar el compromiso, responsabilidad e implicación del equipo docente, facilitadores y profesionales implicados en el desarrollo.

- Implementar estrategias de captación de alumnos.

- Poner en práctica el curso y realizar un seguimiento continuo de la experiencia

\section{Desarrollo de la innovación}

La experiencia que describimos se encuadra en un proyecto europeo, dirigido a la creación y desarrollo de cursos MOOC, titulado "Elearning Communication Open-Data: Massive Mobile, Ubiquitous and Open Learning. (ECO)", en el que participan universidades y empresas europeas y latinoamericanas. Básicamente, el proyecto, iniciado en el año 2014, ha generado un escenario de trabajo colaborativo para el desarrollo y análisis de cursos MOOC a través de la plataforma ECO (http://ecolearning.eu/) en la que se alojan, a su vez, dichos cursos. Asimismo, el proyecto ECO viene desarrollando una significativa tarea de sensibilización de los beneficios de los recursos educativos abiertos, tanto a nivel institucional como social, mediante la difusión de las experiencias en diferentes medios y SNS.

En este marco de trabajo, durante el curso académico 2014/2015, un grupo de investigadores de la Universidad de Cantabria, Universidad de Oviedo y UNED ha 
Análisis de las concepciones pedagógicas y comunicativas de un curso MOOC

diseñado y desarrollado el curso "Innovación educativa y desarrollo profesional. Posibilidades y límites de las TIC", cuyo análisis presentaremos en los próximos apartados. Una síntesis del diseño del curso queda recogida en la tabla 1:

Tabla 1. Diseño pedagógico "Innovación educativa y desarrollo profesional. Posibilidades y límites de las TIC

\begin{tabular}{|c|c|c|}
\hline Bloque & Objetivos y contenidos & Actividades \\
\hline $\begin{array}{l}\text { Semana 1: } \\
\text { ¡Bienvenida! Presentación y } \\
\text { expectativas }\end{array}$ & $\begin{array}{l}\text { Tomar contacto con el entorno de la } \\
\text { plataforma ECO. Primeras interacciones } \\
\text { entre participantes. }\end{array}$ & \begin{tabular}{lccc} 
Actividades & & \multicolumn{2}{c}{ colaborativas } \\
desarrolladas en el & foro de & la \\
plataforma & ECO & $y$ & en \\
Google+/YouTube. & &
\end{tabular} \\
\hline $\begin{array}{l}\text { Semana 2: } \\
\text { Agitando } \quad \text { ideas } \quad \text { sobre } \\
\text { innovación }\end{array}$ & $\begin{array}{l}\text { Identificar ideas previas sobre innovación } \\
\text { educativa con TIC. Analizar el imaginario } \\
\text { sobre las potencialidades de las TIC en } \\
\text { educación. Conocimientos previos sobre la } \\
\text { temática trabajada. Introducción de ideas } \\
\text { básicas sobre el contenido del curso. }\end{array}$ & $\begin{array}{l}\text { Cuestionario de autoevaluación. } \\
\text { Tareas colaborativas desarrolladas en } \\
\text { el foro de la plataforma ECO y en } \\
\text { Google+/YouTube. }\end{array}$ \\
\hline $\begin{array}{l}\text { Semana 3: } \\
\text { Alumbrando el cambio }\end{array}$ & $\begin{array}{l}\text { Diferenciar los conceptos sobre cambios } \\
\text { en los contextos educativos. Identificar } \\
\text { factores que facilitan o dificultan los } \\
\text { procesos de innovación. Contenidos: } \\
\text { conceptos básicos: innovación, cambio, } \\
\text { reforma, etc.; elementos que facilitan las } \\
\text { innovaciones educativas con TIC; aspectos } \\
\text { que dificultan la innovación educativa con } \\
\text { TIC. }\end{array}$ & $\begin{array}{l}\text { Cuestionario de autoevaluación. } \\
\text { Tareas colaborativas desarrolladas en } \\
\text { el foro de la plataforma ECO, en } \\
\text { Facebook y en Twitter. }\end{array}$ \\
\hline $\begin{array}{l}\text { Semana 4: } \\
\text { Innovación } \quad \text { educativa } \quad \text { y } \\
\text { desarrollo } \\
\text { docente }\end{array}$ & $\begin{array}{l}\text { Entender algunas estrategias que permiten } \\
\text { el desarrollo profesional docente a través } \\
\text { de la utilización de TIC. Contenidos: } \\
\text { desarrollo profesional docente; formación } \\
\text { permanente; comunidades de prácticas; } \\
\text { grupos de docentes; andamiaje. }\end{array}$ & $\begin{array}{l}\text { Cuestionario de autoevaluación. } \\
\text { Tareas colaborativas desarrolladas en } \\
\text { el foro de la plataforma ECO, en } \\
\text { Facebook y en Twitter. }\end{array}$ \\
\hline $\begin{array}{l}\text { Semanas } 5 \text { y 6: } \\
\text { Dimensiones básicas de la } \\
\text { innovación con tecnología }\end{array}$ & $\begin{array}{l}\text { Definir las principales dimensiones de la } \\
\text { innovación con TIC: curricular, } \\
\text { organizacional y formativa. Contenidos: } \\
\text { dimensión curricular: interdisciplinariedad, } \\
\text { metodologías, etc.; dimensión }\end{array}$ & $\begin{array}{l}\text { Actividad de evaluación por pares } \\
\text { (P2P). Tareas colaborativas } \\
\text { desarrolladas en el foro de la } \\
\text { plataforma ECO, en Facebook y en }\end{array}$ \\
\hline
\end{tabular}




\begin{tabular}{l|l|l}
\hline & $\begin{array}{l}\text { lonanizacional: flexibilización espacio- } \\
\text { tiempo, talleres internivelares, etc.; } \\
\text { dimensión formativa: comunidades de } \\
\text { práctica, grupos de trabajo, etc. }\end{array}$ & \\
\hline Semanas 7 y 8: & $\begin{array}{l}\text { Elaborar proyectos contextualizados de } \\
\text { innovación educativa con TIC, } \\
\text { Elaborando proyectos de } \\
\text { innovación con TIC } \\
\text { durante todo el curso. Fases de los } \\
\text { (P2P). Tareas colaborativas } \\
\text { proyectos de innovación: apartados de un } \\
\text { compartido, cooperación, asignación de de } \\
\text { responsabilidades, recogida de datos, etc. }\end{array}$ & $\begin{array}{l}\text { Twitter. } \\
\text { plataforma ECO, en Facebook y en }\end{array}$ \\
\hline Semana 9: & $\begin{array}{l}\text { Evaluar la experiencia en términos de } \\
\text { organización, contenido, plataforma, } \\
\text { interacción, etc. }\end{array}$ & \\
\hline
\end{tabular}

\section{Resultados}

\subsection{Nivel pedagógico}

La realización de un diseño pedagógico a partir de la fundamentación teórica, el desarrollo de una documentación amplia y detallada y la aportación de recursos complementarios, de libre acceso y online, nos ha permitido organizar intervenciones estratégicamente, evitando, por un lado, que los participantes mantengan la expectativa de interactuar de forma continua y directa con el profesorado, y, por otro, promoviendo un aprendizaje activo y colaborativo, incentivando el diálogo, la participación y la retroalimentación entre los participantes, con el objeto de generar así una comunidad de práctica.

Los objetivos y contenidos del curso, han sido formulados con el propósito de contribuir a generar un contexto para repensar las innovaciones educativas con la utilización de las TIC mediante experiencias contextualizadas que persiguen la sostenibilidad del proceso de cambio a través de estrategias curriculares, organizativas y formativas, capaces de garantizar el desarrollo profesional de los implicados.

Los materiales desarrollados ad hoc para este curso han tratado de detallar tanto la información general del curso y de cada unidad de contenido (estructura, esfuerzo estimado, objetivos, bibliografía, recursos complementarios, instrucciones para la realización de las evaluaciones y tareas, sugerencia de itinerarios, etc.) como el contenido

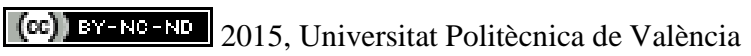

Congreso In-Red (2015) 
trabajado en cada bloque. Dichos recursos han sido preparados de forma contextualizada y presentados en formatos variados, fundamentalmente a través de textos y vídeos accesibles.

Asimismo, se han organizado sesiones sincrónicas online. En algunas ocasiones, estas sesiones han tratado de detallar cuestiones estructurales de cada unidad de contenido al mismo tiempo que aclarar posibles dudas sobre la ejecución de las tareas y evaluaciones propuestas, el uso de los espacios sociales, etc. Dichas sesiones han servido para ampliar la información ofrecida en las guías preparadas ad hoc para el curso, a la vez que para introducir dinamismo e interacción al curso. En estas intervenciones, la comunicación entre el equipo docente y los participantes se ha dado a través de chat o de las redes sociales. Por otro lado, se han desarrollado sesiones de intercambio de experiencias protagonizadas por los participantes del curso y, en algunas ocasiones, por personas expertas en la temática trabajada. En definitiva, con las sesiones virtuales hemos podido aumentar la presencia del profesorado, fortalecer los lazos sociales en la comunidad de aprendizaje, además de servir como "imán" para la captación y retención de participantes.

Por lo que a las actividades se refiere, la planificación prevista de las mismas pretendía acometer un doble reto: garantizar el aprendizaje de los temas trabajados y generar interacción, intercambio y comunicación entre los participantes. Asimismo, algunas actividades han sido sugeridas para que los participantes aportasen materiales producidos por ellos mismos o recursos didácticos e informativos (textos, vídeos, enlaces, eventos, etc.) que pudieran ampliar el contenido propuesto en el curso.

Finalmente, merece destacarse las evaluaciones Peer-to-Peer (por pares), donde los participantes son los responsables de producir materiales, evaluar y dar feedback a los compañeros y compañeras, además de generar debate en torno al tema propuesto en la tarea, sus aplicaciones prácticas en entornos profesionales, etc. Junto a ello, mencionar el lugar que ha ocupado la constante evaluación de proceso a través del seguimiento realizado mediante la sistemática distribución de mensajes (vídeo, audio o texto) de retroalimentación, recogiendo el esfuerzo y logros alcanzados hasta el momento.

En base a lo expuesto, las estrategias pedagógicas analizadas han permitido proporcionar un aprendizaje colaborativo, promoviendo la creación de comunidades de aprendizaje y práctica en las cuales los participantes, en colaboración con el equipo docente, construyen el conocimiento de forma colectiva, intercambiando experiencias, aportando información, sugiriendo recursos didácticos abiertos e incluso evaluando a los demás participantes.

\subsection{Nivel comunicativo}

Para entender las estrategias comunicativas usadas en el curso que aquí presentamos, es importante matizar que el proyecto ECO ha sentado unas bases conceptuales y pedagógicas 
huyendo de algunos convencionalismos de los cursos MOOC. Así pues, los cursos del proyecto ECO son del tipo sMOOC donde la "s" significa social.

Nuestro curso alcanza tres niveles comunicativos a través de:

- Materiales y recursos producidos y sugeridos por el equipo docente (multiformato, abiertos y en línea) y materiales producido o sugerido por los participantes;

- Comunicación entre equipo docente y facilitador y participantes;

- Comunicación entre los participantes (comunidad de práctica y aprendizaje).

En primer lugar, las estrategias del curso están centradas en el desarrollo de recursos, fundamentalmente actividades, que proporcionan y estimulan una interacción constante, dinámica y democrática entre los participantes del curso, y entre éstos y el equipo docente. Las actividades propuestas se centran en el intercambio de información a través de los espacios sociales internos de la plataforma (foro, microblogging, mensajería, etc.) y externos (redes sociales como Facebook, Twitter, Google+, etc.). Junto a ello, han sido propuestas actividades de evaluación por pares que, por un lado, estimulan la producción de materiales, publicados en línea y de libre acceso, a partir de las reflexiones y aportaciones de los participantes y, por otro, promueven la interacción, integración y la retroalimentación entre los participantes.

En segundo lugar, la participación activa y retroalimentación constante del alumnado ha permitido el equipo docente del curso, a lo largo del proceso, pudiera generar o readaptar los contenidos, actividades y recursos de acuerdo al perfil y necesidades de los participantes, así como el intercambio horizontal del conocimiento. Para alcanzar dichas metas, la comunicación entre los participantes y entre los participantes y el equipo docente ha sido constante, abierta, dinámica y democrática, y trataba de apoyar, a la vez que retroalimentar, las estrategias metodológicas y pedagógicas del curso.

En definitiva, todo ello ha contribuido al fortalecimiento de la comunicación e interacción entre los participantes del curso, garantizando así la construcción y manutención de una comunidad de prácticas contextualizada, real y aplicada.

\section{Conclusiones}

El análisis realizado ha permitido estudiar las concepciones pedagógicas y comunicativas inherentes al diseño y desarrollo del curso "Innovación educativa y desarrollo profesional. Posibilidades y límites de las TIC”. Planteado bajo el formato de un sMOOC, un MOOC social y sostenible (Aguaded, Vázquez-Cano y Sevillano-García, 2013), el curso ha 
adoptado un formato online, masivo, abierto y no formal, a la vez que altamente potenciador de la interacción y del trabajo colaborativo.

A nivel de las concepciones pedagógicas, hemos logrado un diseño crítico curricular para la creación de un entorno de trabajo que, lejos de asumir un modelo unidireccional y jerárquico a modo de repositorio, ha favorecido la interacción y los conflictos cognitivos entre los participantes, a partir de un diseño más flexible y abierto (De Waard, I. et al, 2011; McLoughlin, 2013; Moya, 2013). A nivel de las concepciones comunicativas, hemos cuestionado y repensado un modelo de diseño para favorecer la comunicación, garantizando la comunicación para el logro de unas metas de aprendizaje compartidas, evitando la infoxicación de contenidos y buscando la funcionalidad de las experiencias para potenciar la interacción entre los participantes (Gillani, 2013).

A continuación sintetizamos las principales conclusiones de nuestro trabajo en función de los objetivos específicos descritos en el diseño de la experiencia:

- El análisis bibliográfico ha favorecido la potenciación de una visión integral y abierta de la formación permanente del trabajo, lo que nos ha permitido una adaptación continua al contexto de aprendizaje y la reconstrucción conjunta del conocimiento.

- Para poder conseguir un diseño dirigido a promover e incentivar la comunicación entre los participantes, ha sido necesario repensar las actividades para garantizar su contextualización y funcionalidad.

- La adopción de un enfoque curricular superador de las limitaciones de un modelo tecnocentrista nos ha permitido orientar el desarrollo del curso así como el uso las herramientas y recursos (internos y externos) para favorecer la confrontación de perspectivas entre los participantes y la interiorización autónoma de los contenidos en torno a las innovaciones educativas a través de las TIC.

- La negociación lograda en el diseño y desarrollo del curso entre el equipo docente y facilitador, desde una racionalidad crítica curricular, y un modelo comunicativo EMIREC han potenciado la gestión de los canales de comunicación por parte del equipo completo de trabajo así como el logro de un compromiso y responsabilidad compartidos.

- Se ha dinamizado la captación de participantes a través de diversas estrategias: difusión a través de las SNS, utilización de los canales internos de distribución de la información de las universidades participantes, difusión a través de medios de comunicación y establecimiento de vínculos con otras instituciones formativas. Asimismo, la priorización de experiencias de formación, en las que se atiende la diversidad, ha permitido generar itinerarios de aprendizaje personalizados. 
- La realización de un seguimiento continuo ha permitido introducir las mejoras pertinentes, realizando los ajustes para favorecer la interacción entre los participantes y la potenciación de las acciones para la creación de significados comunes en torno a la innovación. Junto a ello, se han implementado estrategias evaluativas, potenciando la evaluación del proceso y la reflexión sobre el aprendizaje.

Finalmente, en virtud del seguimiento realizado y la detección de las principales dificultades, hemos podido plantear alternativas con el objeto mejorar las siguientes ediciones del curso, tales como el incremento de sesiones virtuales realizadas de forma síncrona en cada bloque del curso, la mejora del seguimiento de la actividad de los participantes, las modificaciones en las actividades para facilitar la construcción de itinerarios personales o la búsqueda de hilos conductores de conversaciones on line para evitar la dispersión en el uso de las herramientas.

\section{Referencias}

Aguaded, J.I., Vazquez-cano, E. y Sevillano-García, M.L. (2013). MOOCs, ¿Turbocapitalismo de redes o altruismo educativo? Hacia un modelo más sostenible. En SCOPEO INFORME ${ }^{\circ} 2$. MOOC: Estado de la situación actual, posibilidades, retos y futuro. (págs. 74-90) URL http://scopeo.usal.es/wpcontent/uploads/2013/06/scopeoi002.pdf. (Visitado 2014-02-12).

Cloutier, J. (2010). Historia de la comunicación. En R. Aparici (coord.). Conectados en el ciberespacio. (págs. 37-44). Madrid: UNED.

DeWaard, I., et al. (2011). Using mLearning and MOOCs to Understand Chaos, Emergence, and Complexity in Education. The International Review of Research in Open and Distance Learning, 12(7) URL http://www.irrodl.org/index.php/irrodl/article/view/1046/2026 (Visitado 2014-0218).

Dezuanni, M. y Monroy-Hernández, A. (2012). "Prosumidores interculturales”: la creación de medios digitales globales entre los jóvenes. Comunicar. Revista Científica de Educomunicación, 38(XIX), 59-66.

Fernández-Díaz, E., Rodriguez-Hoyos, C. y Fueyo, A. (2014). Concepciones pedagógicas y comunicativas de los MOOCs: Estado del arte y prospectiva en el marco de un proyecto europeo. III Workshop Internacional sobre creación de MOOC con anotaciones multimedia. Universidad de Málaga. 
Análisis de las concepciones pedagógicas y comunicativas de un curso MOOC

Flores, J. V. et al. (2013). Los MOOCs: Una revolución para la transición a la Sociedad del Aprendizaje. En SCOPEO INFORME N². MOOC: Estado de la situación actual, posibilidades, retos y futuro. (págs. 92-104). URL http://scopeo.usal.es/wpcontent/uploads/2013/06/scopeoi002.pdf.

Gea, M. y Montes, R. (2013). abiertaUGR, la formación abierta basada en comunidades online de aprendizaje. En SCOPEO INFORME No2. MOOC: Estado de la situación actual, posibilidades, retos y futuro. (págs. 122-138) URL http://scopeo.usal.es/wp-content/uploads/2013/06/scopeoi002.pdf. (Visitado 201402-12).

Gillani, N. (2013). Learner Communications in Massively Open Online Courses. OxCHEPS Occasional Paper 53 URL http:// oxcheps.new.ox.ac.uk/MainSite\%20pages/Resources/OxCHEPS_OP53.pdf (Visitado 2014-02-18).

Mackness, J., Mak, S. y Williams, R. (2010). The Ideals and Reality of Participating in a MOOC.

URL http://www.lancs.ac.uk/fss/organisations/netlc/past/nlc2010/abstracts/PDFs/Mackn ess.pdf (Visitado 2014-02-18).

McLoughlin, C. (2013): The pedagogy of personalised learning: exemplars, MOOCS and related learning theories. World Conference on Educational Multimedia, Hypermedia and Telecommunications, Jun 24, in Victoria, Canada.

Montero, I. y Viñuales, J. (2013). Difundi y OpenMOOC. Desarrollando un modelo de negocio basado en servicios MOOC y sobre una plataforma de software libre. En SCOPEO INFORME N²2. MOOC: Estado de la situación actual, posibilidades, retos y futuro. Pág. 140-155. URL http://scopeo.usal.es/wpcontent/uploads/2013/06/scopeoi002.pdf (Visitado 2014-02-12).

Moya, M. (2013). La Educación encierra un tesoro: ¿Los MOOCs/COMA integran los Pilares de la Educación en su modelo de aprendizaje on-line? En SCOPEO INFORME N². MOOC: Estado de la situación actual, posibilidades, retos y futuro. Pág. 157-172. URL http://scopeo.usal.es/wpcontent/uploads/2013/06/scopeoi002.pdf. (Visitado 2014-02-12).

O'Toole, R. (2013) Pedagogical strategies and technologies for peer assessment in Massively Open Online Courses (MOOCs). Discussion Paper. University of Warwick, Coventry, UK: University of Warwick. (Unpublished)

Tapscott, D. (2009). Grown Up Digital. How the Net Generation Is Changing Your World. McGraw-Hill: New York. 